$5-12-2006$

\title{
Group IV Solid State Proposals for Quantum Computation
}

Petru S. Fodor

Cleveland State University, p.fodor@csuohio.edu

J. Levy

Follow this and additional works at: https://engagedscholarship.csuohio.edu/sciphysics_facpub

Part of the Physics Commons

How does access to this work benefit you? Let us know!

Publisher's Statement

This is an author-created, un-copyedited version of an article accepted for publication in Journal of Physics: Condensed Matter. The publisher is not responsible for any errors or omissions in this version of the manuscript or any version derived from it. The Version of Record is available online at 10.1088/0953-8984/18/21/S03

\section{Repository Citation}

Fodor, Petru S. and Levy, J., "Group IV Solid State Proposals for Quantum Computation" (2006). Physics Faculty Publications. 202.

https://engagedscholarship.csuohio.edu/sciphysics_facpub/202

This Article is brought to you for free and open access by the Physics Department at EngagedScholarship@CSU. It has been accepted for inclusion in Physics Faculty Publications by an authorized administrator of

EngagedScholarship@CSU. For more information, please contact library.es@csuohio.edu. 


\title{
Group IV solid state proposals for quantum computation
}

\author{
P S Fodor and J Levy
}

\begin{abstract}
The discovery of the quantum factorization algorithm more than a decade ago triggered intense interest in exploring possible physical realizations of quantum computers. Among the many solid state proposals, electron and nuclear spins in $\mathrm{Si}$ and group IV related materials have long coherence times and the capability of state preparation, gating and read-out using electric, magnetic and optical fields. Proposals involving silicon seek to take advantage of an existing mature technology and the implicit promise of scalability from solid state materials. Nevertheless, building such quantum systems depends in many cases on the development of fabrication techniques with nearly atomic precision. Managing decoherence, initialization and read-out in any quantum computer remains a daunting task. In this review we summarize proposals and recent developments relevant to the possible realization of a quantum computer constructed out of $\mathrm{Si}$ (or group IV) materials.
\end{abstract}

\section{Introduction}

The idea of using quantum states as the basis for the implementation of information processing traces its origin to Feynman [1], who recognized the challenges involved in simulating the behaviour of quantum mechanical systems on conventional computers. For a quantum system consisting of $n$ two-level subsystems (such as spin-1/2), the number of coefficients $D$ needed to describe a quantum state scales exponentially with $n$ (i.e., $D \sim 2^{n}$ ). Even for modest numbers of qubits such as $n=100$, a complete representation of a quantum system using classical hardware is infeasible due to lack of memory. By analogy with the Church-Turing thesis [2] which states that any computer can 'efficiently' simulate any other computer, it is reasonable to expect that a suitably formulated quantum version exists, i.e., that quantum computers should be able to efficiently simulate quantum systems [3].

From a practical point of view, even if the idea of quantum computation is viewed as more of a curiosity, the end of Moore's law scaling [4], will probably lead to more radical design changes in computer architecture that are becoming aligned with issues currently faced by 
designers of quantum computing architectures. As the on-chip transistor sizes reach sub-10 nm dimensions, the computer hardware components will inherently show quantum behaviour, and the computational process will be described by the quantum mechanics laws rather than by the classical physics ones. Being influenced by quantum effects does not by itself enable quantum calculations; rather, the precise control of quantum states (in addition to other requirements discussed below) is what distinguishes a quantum computing architecture from a classical computing architecture increasingly dominated by quantum effects.

As will be discussed below, any practical implementation of the theory of quantum computation faces enormous challenges. The use of quantum based computational algorithms has undeniable advantages in dealing with problems previously considered exponentially difficult by the computer science community. The first quantum algorithm is attributed to Deutsch and Jozsa [5], and was designed to determine if a binary-valued function $f\left(x_{1}, x_{2}, \ldots, x_{n}\right)$ is constant or balanced. On a computing machine obeying the classical laws of physics this computational task would require, in the worst case scenario, a minimum of $2^{n-1}$ iterations, but for the quantum algorithm a single evaluation of $f$ is sufficient to produce a correct answer. Even if the problem of deciding if a function is balanced or not was relatively simple, it proved that there existed a class of computational problems for which quantum algorithms provide more efficient solutions.

Probably the most important contribution to the nascent field of quantum computation was by Peter Shor, who in 1994 introduced his quantum algorithm for the prime factorization of numbers [6]. Shor showed that a quantum computer can greatly outperform its classical counterpart in factorizing large integers (see [7] for a comprehensible review of Shor's algorithm and its implications for modern cryptography). While for a classical computer the time required to factorize an $n$-bit integer scales as $\exp \left(n^{1 / 3} \log _{2 / 3} n\right)$. However, using Shor's algorithm on a quantum computer, the time scales algebraically with the bit size, with the possible polynomial scaling depending on the optimization for the minimization of the qubit number or computational time [8]. The extreme difficulty in factorizing large numbers forms the basis for one of the main encryption schemes based on public key distribution, i.e. the RSA (River, Shamir, and Adelman) encryption scheme [9] which would be made ineffective with the successful implementation of Shor's algorithm on a quantum machine. Shor's algorithm has thus shown that quantum computers can have relevant practical applications beyond being testbeds for the laws of quantum mechanics and simulating quantum mechanical systems.

Another famous algorithm comes from Grover [10], who showed how a quantum database only requires $\sqrt{ } N$ queries for reverse look-up, where $N$ is the number of entries in the database. On average, any ordinary database requires $N / 2$ queries to perform the same task. While the speedup is less dramatic than for Shor's algorithm, the distinction is nevertheless remarkable. A burgeoning 'quantum computer science' field has emerged [11] in which the complete structure of quantum computers has yet to be fully realized.

\section{Quantum computing requirements}

The discovery of Shor's and Grover's quantum algorithms spurred a dramatic increase in the experimental research dedicated to the design and fabrication of the two basic blocks of any quantum circuit: the qubits (registers of information) and the quantum gates (sets of unitary transformations) used to manipulate the qubits [12]. The necessary qualities of any successful practical quantum computer have been summarized by DiVincenzo [13] in five basic requirements:

(1) There must be a scalable quantum system (a two-level system for example) in which the bit of information can be encoded. 
(2) The system should be easily initialized to an initial and well-defined state prior to every new computation.

(3) Whatever the specifics of the quantum system are, the encoded bit should be only weakly coupled to the environment, to reduce decoherence effects and preserve its quantum information for a sufficiently long time to allow computation.

(4) The individual bits and also the interaction between them have to be manipulated in a controlled way, by the implementation of universal single-bit and multi-bit gates. Regarding the multi-bit gates, it is now known that special kinds of two-bit quantum operations, such as the 'controlled-NOT' [14] and ' $\sqrt{ }$ SWAP' gates [14, 15], can be used together with the single-bit operations as the basic blocks for the implementation of any quantum computation operation. With respect to requirement (c), the gate operation time has to be much shorter (typically by $10^{5}$ times) than the decoherence time $T_{2}$ of the qubit.

(5) It must be possible to measure quantum bits with high accuracy.

For the completeness of the functionality treatment of the quantum computer, the five requirements regarding only the computation in the quantum processor can be completed by adding two networkability criteria, addressing the communication issues during the computational process. The controllable transfer of quantum information between different parts of the quantum computer requires:

(6) the ability to convert qubits stored at specific locations into 'flying' qubits and,

(7) the ability to transmit the 'flying' qubits along predetermined interconnections. i.e. buses, throughout the quantum computer.

Naturally, such extremely demanding requirements on any practical implementation of the quantum computation theory was met with scepticism by some researchers $[16,17]$, who argued that difficulties associated with maintaining coherence in large quantum systems will prevent construction of useful quantum computers, i.e., those with a sufficient number of qubits and gates to really compete with classical computers. Designing qubits that couple strongly and controllably to other qubits but not to other degrees of freedom is particularly difficult.

In this context an equally important breakthrough was made: the development of quantum error correction codes (QECC) [18] and the existence of error thresholds for fault-tolerant quantum computing architectures $[19,20]$. With the assumption that the errors affecting individual qubits are independent, Preskill [20], showed that an error rate of $10^{-6}$ per gate is sufficient to permit the implementation of fault correction codes for a particular class of quantum computing architectures. This threshold sets up a corresponding requirement on the lower limit of gating operations to be performed during the decoherence time of a qubit. Nevertheless, this estimate is very conservative [21] and depends on the details of the architecture; with active quantum state stabilization error threshhold values of $10^{-5}$ are possible [22]. The very existence of QECC might seem surprising: how to repair an unknown error in a state that cannot itself be measured? As DiVincenzo and Loss discuss in [23], Landauer's early criticism [17] seemed even more damning: the Schrödinger equation is a continuous differential equation, and should therefore be subject to a theorem of Shannon which states that errors in analogue signals cannot be corrected [24]. Peter Shor discovered how error correction based on quantum entanglement can provide a way out of this dilemma, and paved the way for scores of theorists and experimentalists to devise proposals for implementing real quantum computers.

The first quantum computation experiments involved nuclear magnetic resonance [25], and later cavity quantum electrodynamics [26], harmonic ion traps [27], and optical approaches [28] soon followed. All of these approaches contain the fundamental ingredients for the implementation of simple quantum algorithms. Less clear is how these approaches could 
realistically be applied to much larger numbers of qubits and qugates. The issue of scalability to large quantum systems looks to be most easily addressed by solid state proposals, given the already extremely successful approach to the fabrication of modern microprocessors. In 1998, Loss and DiVincenzo proposed using electron spins confined in semiconductor quantum dots as qubits for quantum information processing [29]. While the proposal referred to a specific representation of the quantum processor hardware, their framework was quite general and has been applied by others [30-33] to almost every two-level spin system (electronic or nuclei) localized in a solid state environment. The one-bit operations on electron spins localized at each individual dot are performed by electrically controlling their coupling with a neighbouring ferromagnetic dot. Similarly, two-qubit operations are performed by electrically pulsing the exchange interaction $J$ between electrons confined at different semiconductor quantum dots. During the two-qubit operation the time-dependent Heisenberg exchange coupling: $H(t)=J(t) \vec{S}_{1} \cdot \vec{S}_{2}$ induces a unitary transformation:

$$
U=\exp \left[-\mathrm{i} \int_{0}^{T} H(t) \mathrm{d} t / \hbar\right]
$$

If the exchange interaction is controlled such that $\int_{0}^{T} J(t) \mathrm{d} t / \hbar=\pi / 2$, often called a 'square-root swap' or SWAP ${ }^{1 / 2}$ operation, then one has the ability to entangle two qubits. This operation, combined with single-qubit operations, is sufficient for universal quantum gating [34]. In their proposal, the final state measurement is achieved with an accuracy of $75 \%$ through electron tunnelling into a supercooled paramagnetic dot, the nucleated spontaneous magnetization direction being correlated with the spin state of the electron qubit. An alternative is spin-dependent tunnelling into a third dot where the electron presence is determined using a sensitive electrometer. The benefit of this spin-valve approach is twofold, allowing 100\% measurement accuracy and also a more efficient initialization of the system without reliance on thermalization in magnetic fields.

Because of the large number of existent solid state proposals for quantum computation it would be difficult to give an exhaustive survey of all the solid state approaches. Consequently, this review will discuss only the proposals involving the manipulation of electronic and nuclear states in group IV based materials, with a particular emphasis on those involving silicon.

\section{Silicon for quantum computing}

Considering the fact that silicon is the 'workhorse' of modern conventional computer electronics, it is not surprising in retrospect that it was considered early on as a possible material system for a solid state quantum computer [30]. Proposals that utilize silicon generally seek to take advantage of three particular characteristics of silicon: (a) weak spin-orbit coupling in silicon; (b) the possibility of obtaining isotopically pure $\mathrm{Si}^{28}$ crystals; and (c) a vast technological infrastructure associated with the silicon industry.

Most of the proposals involving silicon as the material of choice employ the electron spins or donor $I=1 / 2$ nuclear spins to represent quantum information. In semiconductors, the major sources of decoherence for the electron spin are due to coupling to the lattice phonons through the spin-orbit interaction, and coupling to nuclear spins through the hyperfine interaction. These interactions are described by the following Hamiltonian:

$$
H=H_{\mathrm{so}}+H_{\mathrm{hf}}=\underset{2 m^{2} c^{2}}{\hbar}(\nabla V(\vec{r}) \times \vec{p}) \cdot \vec{S}+\sum_{i} A_{i} \vec{S} \cdot \vec{I}_{i}
$$

where $V(\vec{r})$ is the potential in which the electrons move, $\vec{p}$ is the momentum operator, $\vec{S}$ is the electron spin operator, $A$ is the hyperfine interaction strength and $I$ is the nuclear spin 
operator. For the case of nuclear spins, the main decoherence mechanism, besides the hyperfine interaction, is the long range dipole-dipole interaction between different nuclei. The dipolar term can be expressed as

$$
H_{\text {dip }}=\sum_{i, j} \vec{I}_{i} D_{i j} \cdot \vec{I}_{j}
$$

where $D_{i j}$ is the dipolar interaction tensor.

In $\mathrm{Si}$ these spin decoherence times, both for the electrons and nuclei, can be quite long compared to other semiconductor systems, due to the weak spin-orbit coupling $(|g-2|<$ $3 \times 10^{-3}$ ). At low temperatures, changes in the Larmor frequency determined by the hyperfine interactions remain the primary sources of decoherence [35]. The Bohr radius of an electron bound to a donor is about $20 \AA$ [36], so the number of nuclear spins that couple with the donor spin is about $\sim 100$, for natural silicon with an isotopic concentration of $4.67 \% \mathrm{of} \mathrm{Si}^{29}$ isotopes. Thus, for commercially available isotopically purified $\mathrm{Si}^{28}$ (99.9\%) [9] the number of $\mathrm{Si}^{29}$ nuclear spins coupled with a donor can be further reduced to about $\sim 3$. From this perspective group IV semiconductors present a definite advantage when compared with group III-V semiconductors, which do not have spin-zero nuclear isotopes. In fact, natural Si contains about $95 \%$ spinless $\mathrm{Si}^{28}$ or $\mathrm{Si}^{30}$ isotopes; for naturally abundant carbon the spin-zero isotope is $99 \% \mathrm{C}^{12}$; these values can be improved by standard purifying techniques. Gordon and Bowers' spin-echo measurement from 1958 of the transverse decoherence times for the donor electron spins in isotopically enriched $\mathrm{Si}^{28}$ yielded values for $T_{2} \sim 0.5 \mathrm{~ms}$ at temperatures $<10 \mathrm{~K}$ [35]. More recently, electron spin decoherence times for low donor concentration as large as $T_{2}=60 \mathrm{~ms}$ have been extrapolated for isolated spins [37]. These values are five orders of magnitude larger than $T_{2}^{*}$ values reported for III-V materials [38, 39], where it has been confirmed many times over that nuclear spins provide the dominant mechanism for electron spin decoherence. Regarding the nuclear spins, double-resonance experiments have shown for low donor $\mathrm{P}^{31}$ nuclear spins that the decoherence times can exceed ten hours at $T=1.5 \mathrm{~K}$ [40].

Charge qubits associated with donor atoms in Si have also been considered for quantum computation [41]. One of their great advantages is the fast electrical gating operation times which can approach 50 ps [42], and also the potential for using single-electron transistors (SET) [43] and field effect transistors (FET) [31] for measuring single charge states. As will be discussed in section 5, even for spin based approaches most read-out schemes are based on spin-to-charge conversion schemes. One drawback of charge based qubits is the strong coupling to other stray charges that may exist nearby, which induces large decoherence effects [44]. Only recently it has been demonstrated that for a specially designed capacitively coupled charge-qubit the decoherence times can be much larger than the typical values of a few nanoseconds [45]; furthermore, dipolar interactions complicate the gating mechanism in twoqubit interactions. Nevertheless, the following discussion regarding the placement of impurities donors in a Si host is relevant for both the spin and charge based approaches for the use of Si as the base material for quantum computation.

\section{Nuclear spins in silicon for quantum computing}

\section{Nuclear spins of shallow donor impurities in Si}

In a search to take advantage of the well-decoupled nuclear spin states of donors in $\mathrm{Si}$, Kane [30] proposed the use of the nuclear spins of $\mathrm{P}^{31}$ shallow impurities in isotopically pure zero-nuclear-spin $\mathrm{Si}^{28}$, as a representation for the qubit (figure 1). The $\mathrm{P}^{31} I=1 / 2$ nuclear spins are decoupled from the environment in the spin-zero crystalline lattice with the exception of the hyperfine interaction with the associated weakly bound electrons in a non-degenerate 

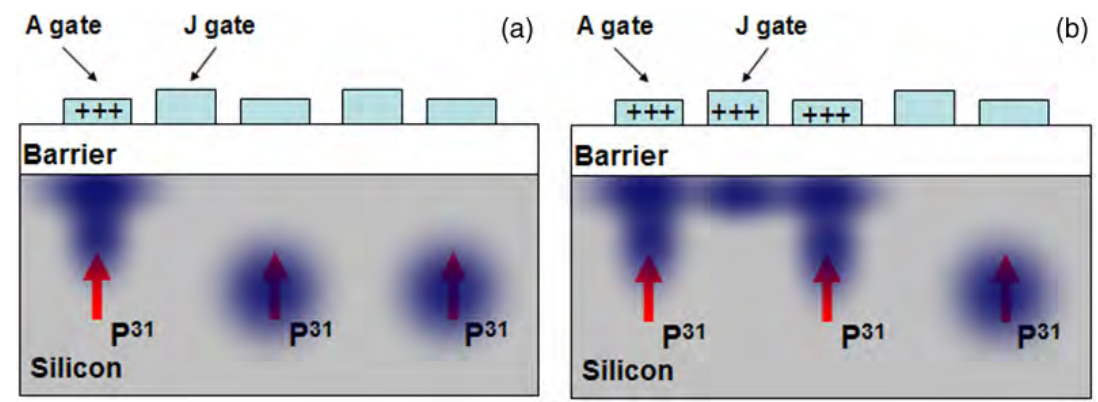

Figure 1. Schematic drawings of (a) single-bit operation through electrostatic control over the hyperfine interaction using an A gate; (b) two-bit electron exchange mediated operation on two nuclear spins associated with $\mathrm{P}^{31}$ impurities in silicon.

s-type orbital state. While at low temperatures the electron wavefunction is concentrated at the parent donor atom, it still extends a couple of nanometres from the donor nucleus which allows coupling of neighbouring donor nuclear spins through the superexchange interaction mediated by the electron spins. Electrostatic gates, A gates, placed above the impurity donors, control the hyperfine coupling $A$, allowing the donor nuclear spins to be brought in resonance with an external microwave magnetic field. Local control of electron wavefunctions allows for individual nuclear spins to be manipulated in a globally applied field, enabling single-qubit operations. Two-qubit operations are mediated by gates placed in between the $\mathrm{P}^{31}$ impurities, $J$ gates, allowing the controlled overlap of electron wavefunction localized at neighbouring donors, thus controlling the exchange coupling $J$ between neighbouring donors in an on/off fashion.

Throughout the computational process the electrons are required to remain in their lowest energy spin state, imposing requirements on the working temperature and magnetic field. The electrons will be polarized by a dc magnetic field of $B=2 \mathrm{~T}$ at temperatures $T \sim 100 \mathrm{mK}$. Nevertheless, due to the much smaller nuclear magnetic moment, the nuclear spins are not completely polarized. Obtaining an initial high degree of polarization of the nuclear spins represents a problem for all the nuclear spin quantum computation proposals, most of them requiring like the original Kane proposal the interactions with a bath of electrons in a preferential spin state. Besides thermalization in magnetic fields, other avenues have been proposed to achieve the necessary degree of electronic polarization including optical pumping of electron in a single spin state using circularly polarized light (optical orientation) [46], spin refrigeration using a spin filter [43], algorithmic cooling through the redistribution of entropy between two quantum registers, one of which is subsequently discarded [47], magnetic resonance force microscopy [48], and injection of spin polarized electrons from ferromagnetic semiconductors [49].

Since the read-out of the final computational result would involve the measurement of the state of extremely small nuclear magnetic moments, Kane proposed a 'spin-to-charge' conversion scheme similar to that described by Loss and DiVincenzo [29], based on spindependent tunnelling between two donor impurities. A review of the final state measurement problem which is common to all the solid state spin based quantum computation approaches is given in section 5 .

While the Kane proposal contains the basic elements in terms of qubits and gates for the construction of a silicon based quantum computer, its implementation depends upon significant advances in the ability to place individual donors into $\mathrm{Si}$ and register the required electrostatic 


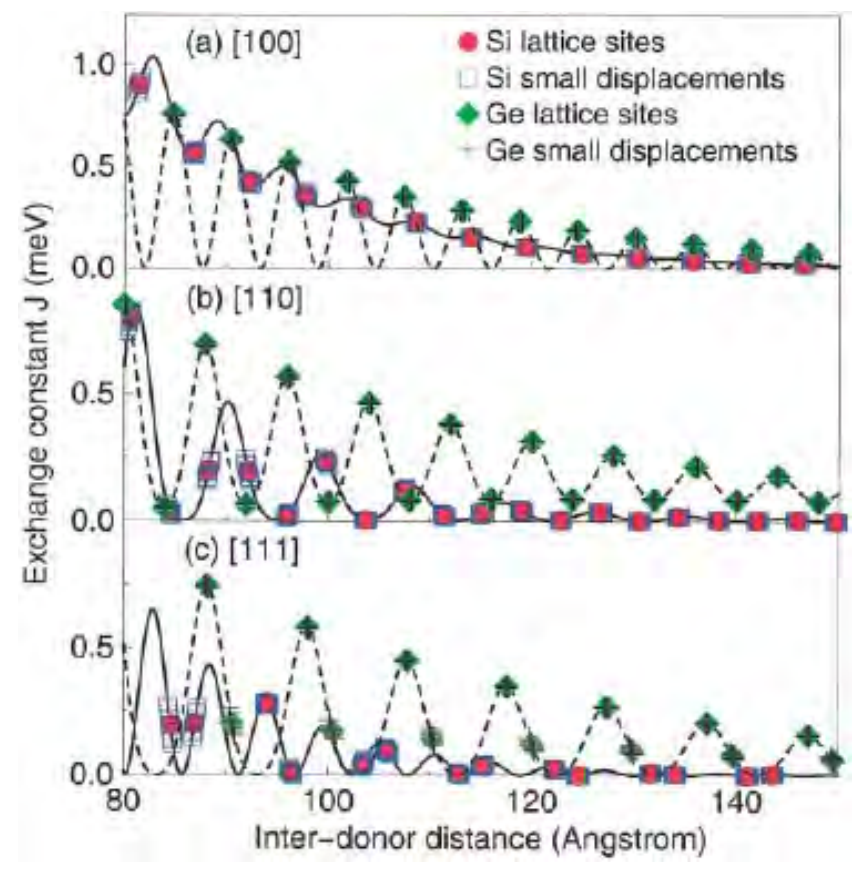

Figure 2. Electron mediated exchange interaction between two donors in Si (solid lines) and Ge (dashed lines). Reprinted with permission from Koiller et al [50], @2002 The American Physical Society.

gates to this individual nuclear spins. Implementation of two-bit operations based on electron mediated superexchange between donor nuclear spins depends exponentially on the separation between donor states, requiring $\sim 20 \mathrm{~nm}$ spacings for reasonable values of the nuclear spin exchange. Despite the uniform nature of the $\mathrm{P}$ donor state, variations in the actual spacings require each gate to be individually calibrated [15]. In fact with respect to the device variability, a more subtle point has been made by Koiller et al $[50,51]$, who pointed out that the six equivalent energy minima in the Si conduction band contribute equally to the wavefunction of the electron trapped at donor atoms. The quantum interference determined by this degeneracy leads to rapid oscillation of the electron mediated coupling between the donor nuclear spins over distances comparable with the lattice constant in Si (figure 2). Nevertheless, it has been noted that this degeneracy can be broken by strain and/or applied electric fields [50, 52].

Consequently, the device variability problem can be addressed only with technologies capable of single-donor placement in $\mathrm{Si}$, with close to atomic resolution. From this point of view two techniques are promising: one involving scanning tunnelling microscopy (STM) manipulation and the other one involving donor implantation with focused ion beams. In the 'bottom-up' approach based on STM lithography [53], hydrogen atoms on a passivated Si surface are removed by scanning a STM tip at high bias voltages. Phosphorus atoms are selectively deposited by absorption of $\mathrm{PH}_{3}$ precursor molecules on areas where bare Si dangling bonds have been exposed due to the removal of the $\mathrm{H}$ atom resist. Controlled incorporation of single-dopant $\mathrm{P}$ atoms on silicon surfaces has been demonstrated with $1 \mathrm{~nm}$ accuracy [54]. This technique, combined with registration markers (figure 3(a)) [55] for final metallic gate alignment or gate patterning with focused ion beams (figure 3(b)) [56], could lead to a robust implementation of the Kane architecture. 


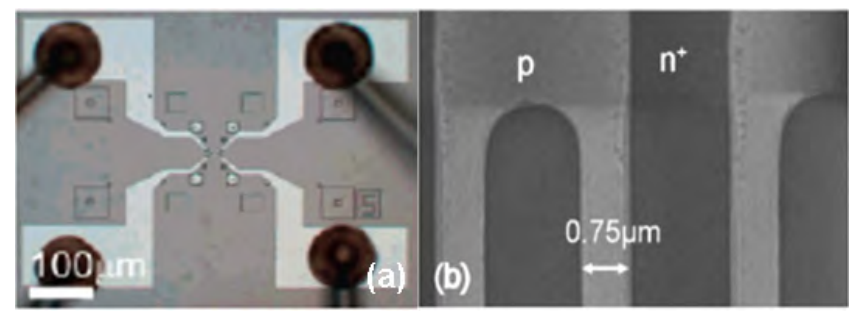

Figure 3. (a) SEM image of an electrically contacted nanoscale Si:P buried device patterned using STM lithography, encapsulated by Si MBE and aligned to registration markers on the surface. Reprinted with permission from Ruess et al [55], (C2004 The American Chemical Society; (b) AFM image of $\mathrm{As}^{+}$implanted lines compatible with $\mathrm{P}$ donor implantation. Reprinted with permission from Shen et al [56], (C2004 American Vacuum Society.

One of the main material issues still remaining is control over the dopant segregation during the epitaxial growth of the capping layers. Recent STM studies showed that for room temperature encapsulation the segregation length of $\mathrm{P}$ in $\mathrm{Si}$ is of the order of $\sim 0.29 \mathrm{~nm}$ [57]. A more precise and reproducible alignment of the nuclear qubits with the corresponding electrostatic gates might be achieved in a 'top-down' approach, in which low energy $\sim 15 \mathrm{keV}$ focused ion beams are used to implant $\mathrm{P}$ donors into $\mathrm{Si}$ after the electrostatic gates and the read-out structures (SET) are fabricated. It is estimated that placement of $\mathrm{P}^{31}$ donors with $\pm 10 \mathrm{~nm}$ accuracy can be realized with high probability [58]. An improvement in accuracy might be achieved using sacrificial resist masks and on-chip integrated detection of single ion implantation events [41].

The demand for the 10-20 nm separations between the donor impurities is relaxed if the coupling between nuclear spins can be mediated by long range interactions. The use of long range dipolar interaction has been suggested [59] with the use of a double- $\pi$-pulse sequence to suppress undesired coupling between qubits that do not participate in the computation. An increase of three times in the allowable separation is estimated at the expense of slower gating times due to the non-exponential suppression of the dipolar interaction, when compared with the exchange interaction.

Privman et al [60] have proposed an architecture in which the nuclear spins are coupled to a highly correlated 2DEG in the quantum Hall regime. In this case the separation of donor nuclei can be as large as $100 \mathrm{~nm}$ for nuclear spins coupled through the localized donor electrons interacting with the delocalized $2 \mathrm{D}$ electrons. While the spin exciton scattering on the impurities is expected to be the dominant dephasing mechanism in these systems, the decoherence time $T_{2}$ will still be in the range of $0.1 \mathrm{~s}$ for a high quality 2DEG [61]. High quality 2DEG structures are also relevant for a more recent update of the Kane proposal [62] in which the multi-bit operations are realized by electron shuttling between donor impurities using electrostatic gates similar to the operation of CCD devices (figure 4). In this proposal the qubit is hydrogen-like with the two-state basis defined by $|0\rangle=(|\uparrow \downarrow\rangle-|\downarrow \uparrow\rangle) / \sqrt{ } 2$ and $|1\rangle=(|\uparrow \downarrow\rangle+|\downarrow \uparrow\rangle) / \sqrt{ } 2$, where the logical qubit states are formed from entangled states between the electron and nuclear spins. Entanglement between two logical qubits is achieved by firstly applying a sufficiently high bias on an A gate to produce ionization of the corresponding qubit. A timed pulse sequence is then applied on the $\mathrm{S}$ gates placed between the impurity donors to bring that electron in the vicinity of the second electron-nuclei spin pair. Coherent transport of electron spins over distance of the order of $100 \mu \mathrm{m}$ has already been demonstrated in semiconductors [63] and offers a solution not only for relaxing the requirement on the small separations between impurity donors, but also provides an avenue for 

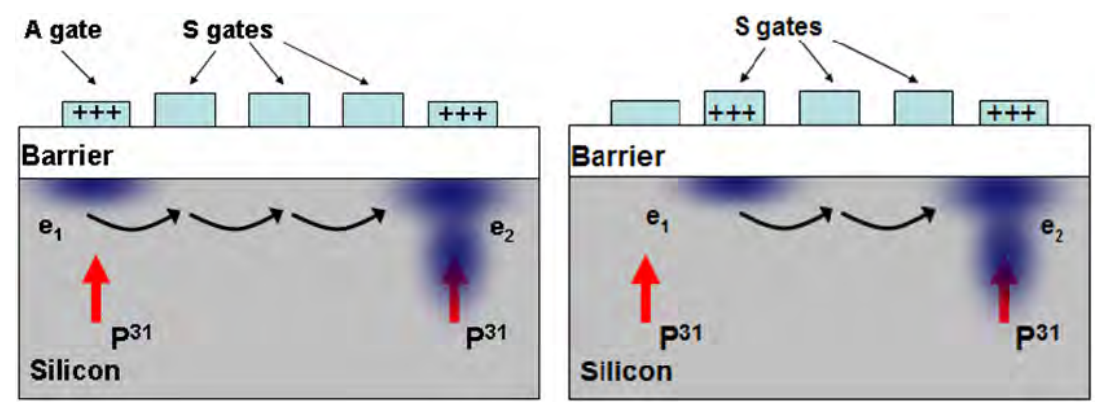

Figure 4. Electron shuttling from one donor impurity to another one by applying a sequence of timed electric pulses on A and S gates respectively.

generating the so-called 'flying qubits' critical for interconnectivity in the quantum computer. Nevertheless, this requires the fabrication of high mobility 2DEG layers in Si in the presence of impurities and compatible with subsequent gating for single-and two-qubit operations. Progress in this direction has been achieved using a hydrogen passivated $\mathrm{Si}$ surface gated through an encapsulated vacuum cavity. Mobilities as high as $\sim 8000 \mathrm{~cm}^{2} \mathrm{~V}^{-1}$ at $4.2 \mathrm{~K}$ have been observed using 2DEG obtained from this technology [64], representing a threefold increase when compared with metal oxide semiconductors structures. Also in a recent extension of this proposal the requirements regarding high gated density have been relaxed by separating the regions in which the quantum information is stored and processed, respectively [65].

\section{$\mathrm{Si}^{29} \mathrm{NMR}$ quantum computer}

In order to circumvent the fundamental challenge in nuclear spin based solid state quantum computers of measuring single nuclear spin states, Ladd et al [66] have proposed a linear chain NMR computer composed entirely of silicon. Spin-1/2 $\mathrm{Si}^{29}$ isotopes deposited as atomic chains on isotopically purified $\mathrm{Si}^{28}(111)-(7 \times 7)$ surface, constitute the two-state basis for performing the quantum computation process (figure 5). Each individual atomic chain is located next to a ferromagnetic strip, such as NiFe alloy or Dy, which produces a strong magnetic field gradient $\sim 1.4 \mathrm{~T}_{\mu \mathrm{m}}^{-1}$. Due to this field gradient, the nuclear spin qubits along the atomic chain become addressable in external magnetic fields due to frequency differences between neighbouring $\mathrm{Si}^{29}$ nuclei of the order of a couple of $\mathrm{kHz}$ [67]. The ferromagnet can also be used to inject spin polarized electrons to initialize the qubits through the hyperfine contact exchange between electron and nuclear spins. Nuclear magnetic resonance is detected using magnetic resonance force microscopy (MRFM) [66] with the device embedded in a bridge cantilever. One major advantage of such an implementation is that for the size of the device envisioned, each particular nucleus has about $10^{5}$ copies in separate atomic chains giving a proportional increase in the signal for the final state read-out. In principle the elimination of unwanted spins, including the electron spin, except during initialization, removes most of the decoherence mechanisms except the dipolar interaction, yielding transverse decoherence times $T_{2}$ of up $25 \mathrm{~s}$ [68]. Reproducible fabrication of atomically straight step edges on $\mathrm{Si}(111)$ and NiFe thin ferromagnetic strips has been recently demonstrated [69] with possible future integration with deposition of $\mathrm{Si}^{29}$ isotopes.

\section{Electron spin proposals}

For quantum computation applications the number of gate operations that can be performed without losing coherence is the most important parameter from the point of view of error 


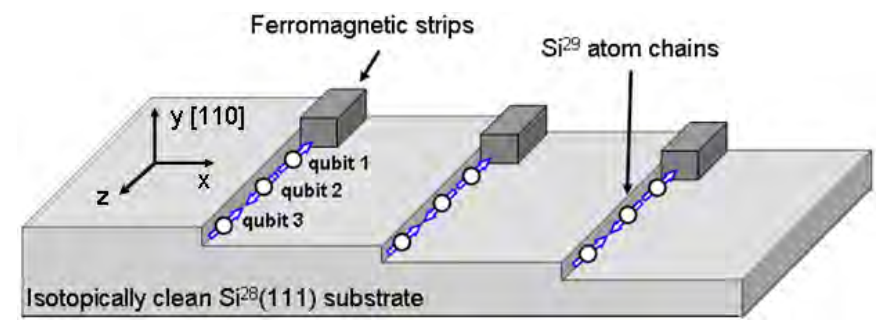

Figure 5. Schematic drawing of a $\mathrm{Si}^{29} \mathrm{NMR}$ quantum computer. Ferromagnetic strips create a field gradient along the atomic chains deposited at terrace edges on $\mathrm{Si}^{28}(111)$ surface.

correction codes. While the extremely long transverse spin decoherence times makes the use of nuclear spins desirable as candidates for qubit representation, their relative small Zeeman splitting limits the clock speed for single-bit operation to about $\sim 75 \mathrm{kHz}$, which is small when compared with values in the $\mathrm{GHz}$ range achievable for electron spin adiabatic gating [70]. The need to exert local control over the Zeeman splitting to perform one-qubit rotations can be eliminated using three or more equivalent spins to represent a single qubit $[71,72]$; the price one pays is the excess hardware as well as an increase in the number of physical gate operations per logical qubit operation. If two inequivalent environments can exist, then it is possible to perform universal quantum gating using only two spins, with negligible increase in computational overhead [73]. The advantage of exchange-only proposals is the increased simplicity in design, with only a single-gate type required for all quantum operations. In exchange-only architectures, most of the proposals based on nuclear spin qubits in Si still rely on precise control of the hyperfine interactions between the electron and nuclear spins both for gating as well as for initialization and read-out of the quantum computer. Consequently, most recent proposals for a $\mathrm{Si}$ based quantum computer have considered the use of electron spin states [31-33] to provide the basis for quantum computation, despite the noticeably shorter spin decoherence times.

\section{Electron spins of shallow donor impurities in $\mathrm{Si}$}

Vrijen et al [31] have considered the use of the spin states of the electrons associated with shallow $\mathrm{P}^{31}$ donors as the qubit representation. Similarly with the nuclear spin proposal the electron wavefunction is shaped by means of voltages applied to metallic gates placed above the donor impurities. Nevertheless, the donor atoms are embedded in a SiGe heterostructure rather than pure Si (figure 6), and the proposal relies heavily on strain and band structure engineering both for the confinement as well as the manipulation of the electrons.

The $\mathrm{P}^{31}$ impurities are embedded in a $\mathrm{Si}_{0.4} \mathrm{Ge}_{0.6}$ layer (D layer) capped with a $\mathrm{Si}_{0.15} \mathrm{Ge}_{0.85}$ layer (T layer). The two regions provide a strong electron $g$ factor contrast, from an isotropic value of 1.998 in the $\mathrm{Si}$ rich D layer to a $g$ factor of 0.823 along the $\langle 111\rangle$ axis in the Ge rich region. Thus the electron spin can be brought in resonance with an external magnetic field through the $g$-factor modulation obtained by electrostatically controlling the electron wavefunction overlap with the two layers $[31,74]$.

Due to the relatively strong spin-orbit coupling, the $g$ factor in Ge is anisotropic, and is determined by the angle $\phi$ between the $\langle 111\rangle$ crystallographic direction and the external magnetic field:

$$
g^{2}=g_{\|}^{2} \cos ^{2} \phi+g_{\perp}^{2} \sin ^{2} \phi
$$




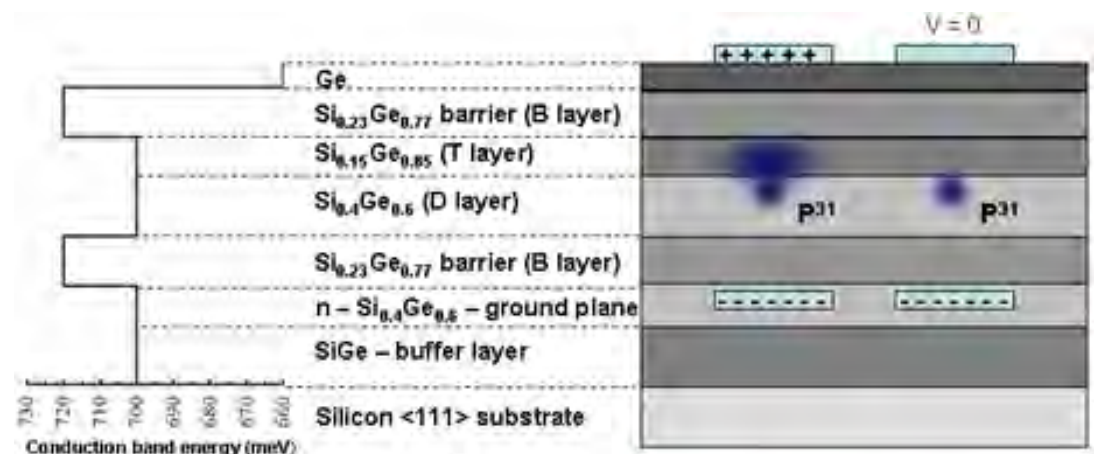

(a)

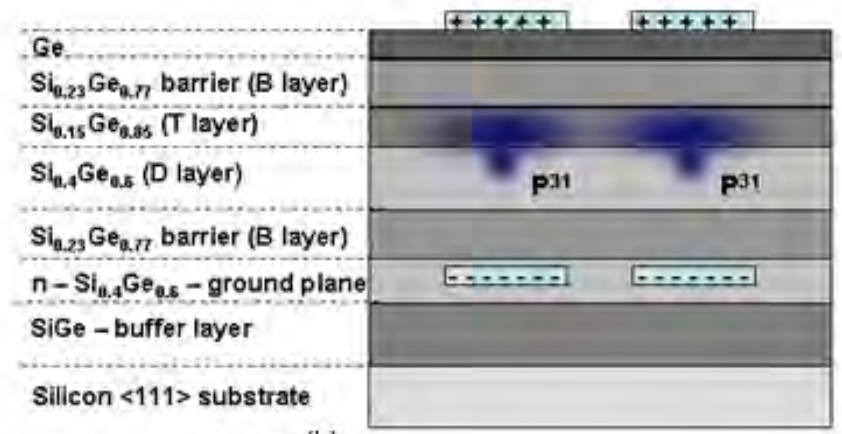

(b)

Figure 6. (a) One-bit operation on the electron spin localized at a $\mathrm{P}^{31}$ donor site in a SiGe heterostructure. (b) Two-bit operation with simultaneous positive voltages applied to metallic gates registered with neighbouring donors (adapted from [31]).

In III-V materials the anisotropic nature of the $g$ factor, which can be regarded as a controllable effective magnetic field, has already been exploited for three-dimensional control of the electron spin [75] and electrically induced spin resonance [76] without the need for microwave magnetic fields, thus providing the basic ingredients for single-bit operations.

As in the Kane proposal, two-qubit operations are performed by applying a sufficiently high positive bias between the top gates and a back gate to induce wavefunction overlap between localized electrons. During this operation the electron wavefunction is strongly peaked in the Ge rich region, where the Bohr radius is about three times larger that in $\mathrm{Si}$, reducing the demands regarding the placement of impurity donors and registering the gates. Also, it is speculated that the use of $\mathrm{Si}_{0.23} \mathrm{Ge}_{0.77}$ barriers (B layers) to confine the spin carriers will allow the Coulomb coupling of the electron with the parent donor to be adjusted on a wide range without the occurrence of ionization, thus allowing the exchange interaction to be turned on or off even for donor separations of $200 \mathrm{~nm}$ [31]. The $J$ gates become redundant since switching between the one-and two-qubit operations can be performed by applying different magnitude bias voltages on the top gates. From a technological standpoint, while the requirements for lateral lithographical accuracy are greatly reduced, placement of single donors while maintaining high quality structures remains a challenge.

One of the primary advantages of using spins states of electron and nuclei associated with impurity donors for quantum computation is the fact that they are naturally identical two-level quantum systems. Also, in the case of the electrons the coupling with their parent donor allows 
the spatial localization of their wavefunction at specific positions that can be registered with electrostatic control gates. However, the strong dependence of the electron exchange energy on the position makes shallow donor based architectures technologically challenging since they require very high accuracy in positioning of individual qubits.

\section{Electron spins in quantum dots}

Early in the development of solid state proposals for quantum computation, quantum dots systems had been envisioned by Loss and DiVincenzo [29] as possible candidates for building the hardware of quantum computers. They provide the necessary localization of electrons on length scales comparable to the electron Fermi wavelength (nanometres to close to a micrometre range) and also exhibit distinct discrete energy spectra due to quantum confinement. The three-dimensional confinement of electrons essentially reduces their coupling with the environment and is believed to suppress some of the decoherence mechanisms that cause spin-flip scattering events [77]. Because quantum confinement for quantum dots is typically weaker than for donor states, the electron wavefunction tends to be more delocalized, thus reducing the demands on the placement of gates. Extensively studied confined systems include those obtained by electrically gating 2DEG [78] and self-assembled quantum dots grown in a Stranski-Krastanov epitaxial growth mode [79]. For III-V semiconductors, precise control over the number of electrons in the dot has been achieved through both lateral [80] and vertical [81] electrical gating. Electrical control over electrons in quantum dots has been recently used to determine lower bounds for the electron spin relaxation times in individual quantum dots, both for electrostatically defined GaAs dots $\left(T_{1} \geqslant 0.85 \mathrm{~ms}\right.$ at $\left.B=8 \mathrm{~T}\right)$ [82] as well as for InGaAs self-assembled dots $\left(T_{1} \geqslant 20 \mathrm{~ms}\right)$ [83]. The more relevant (at least for quantum computation) inhomogeneous spin dephasing time depends not only on the material (i.e., GaAs versus $\mathrm{Si}$ ) but also the local environment and the nature of the bound state. Values ranging from $T_{2}^{*}=10 \mathrm{~ns}$ [84] to $25 \mathrm{~ns}$ [39] have been reported for zero magnetic field using independent methods in III-V systems. For III-V systems, transverse decoherence is dominated by spin-flip processes with nuclear spins [39], consistent with earlier theoretical results [85].

While the experimental study of similar confined structures in group IV materials is far less advanced, the possibility of removing hyperfine interactions through isotopic purification remains a strong motivation for considering group IV semiconductors as hosts for spin based quantum information processing technology. Friesen et al [32] have introduced an implementation of the Loss-DiVincenzo proposal [29] in which the electrons are laterally confined using top electrostatic gates on a SiGe heterostructures (figure 7). The vertical confinement is achieved through band engineering confinement in a strained Si quantum well sandwiched between $\mathrm{Si}_{0.7} \mathrm{Ge}_{0.3}$ layers. The exchange interaction between individual electron spins is pulsed by applying a defined bias voltage on the top gates, while a back gate is used to control the occupancy of each dot in the array. A detailed modelling of a four-bit array has shown that two-bit operations can be performed with a minimum effect on the state of the other qubits in the array. From the fabrication point of view some of the largest issues of the architecture are dislocation free growth to ensure a high quality 2DEG and high quality top Schottky contacts. The success of controlling charge and spin degrees of freedom of electrons in the GaAs/AlGaAs based similar structures was determined in much part by the lack of strain in these heterostructures. On the other hand it is well documented that due to the $4 \%$ lattice mismatch between $\mathrm{Si}$ and $\mathrm{Ge}$, the growth of $\mathrm{Si}_{0.7} \mathrm{Ge}_{0,3}$ on bare $\mathrm{Si}$ occurs with a density of threading dislocations as high as $10^{10} \mathrm{~cm}^{-2}$, significantly reducing the electron mobility [86]. The lattice mismatch strain can be reduced using a strain relaxed buffer layer in which the Ge concentration is ramped to the nominal value of $30 \%$, thus controlling the 


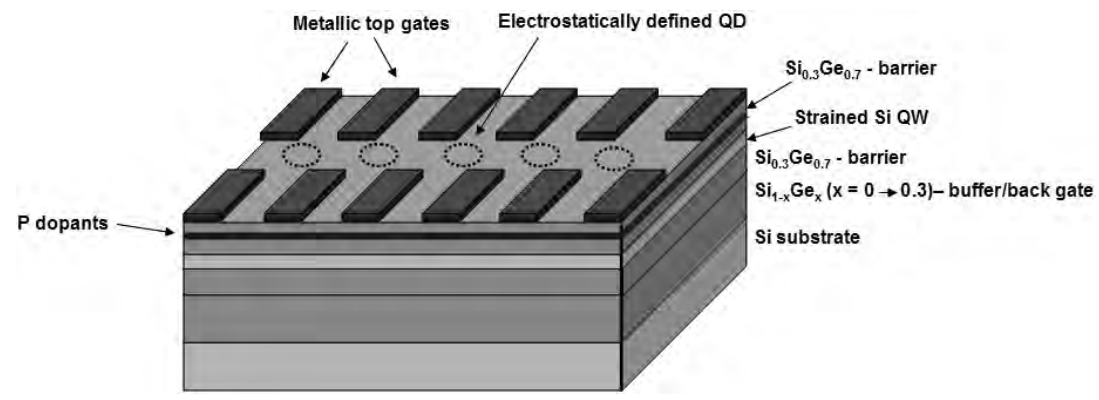

Figure 7. Schematic drawing of an electrostatically defined linear array of quantum dots. The top metallic gates are used both to laterally confine electrons in a strained silicon quantum well and also to control the exchange interaction between electrons trapped at different sites. The electron occupancy number is controlled via back gates below the quantum dot layer.

density of threading dislocations. Recently reported results on structures grown through this technique have demonstrated 2DEG with electron density of $4 \times 10^{11} \mathrm{~cm}^{-2}$ and mobilities of $40000 \mathrm{~cm}^{2} \mathrm{~V}^{-1} \mathrm{~s}^{-1}$ at temperatures $T<4 \mathrm{~K}$ [87].

Lattice dislocations and the proximity of the dopant layer to the metallic gates also affect the quality of the Schottky gates required to laterally confine the 2DEG. Recently the same research group has reported high quality Schottky contacts on SiGe heterostructures using the above-mentioned strain relaxation technique and a hybrid approach involving deposition and etching [88]. The final low leakage of the structure allows the observation of conductance oscillations associated with the Coulomb blockade during the single-electron charging of the quantum dots, demonstrating an important milestone for the use of SiGe heterostructures for quantum computation.

Even with convergent solutions for the material issues facing quantum computation applications, one factor that limits many of the approaches involving electrostatic control is the accuracy and speed with which the exchange interaction can be pulsed using electrical pulses. In order to achieve $10^{5}$ operations during the decoherence time, required for error correction codes, the amplitude stability of the voltage generator has to be within $\delta V / V \leqslant 10^{-2.5}$ over the bandwidth for which gating occurs. Also the jitter in the pulse length of $\delta T \approx 100$ ps limits their operation, assuming typical decoherence times $T_{2}=0.5 \mathrm{~ms}$, to $\mathrm{MHz}$ ranges rather than the required $\mathrm{GHz}$ range.

The use of optical fields rather than electrical pulses for controlling the qubit-qubit interaction is an attractive alternative, given the performance of ultrafast lasers both in terms of amplitude stability and timing accuracy. Timing jitters of less than $240 \mathrm{fs}$ at a bandwidth of $5 \mathrm{GHz}$ are small enough to satisfy the requirements for error corrected quantum computation [89]. Levy [33, 90] has proposed the use of a silicon based oxide-semiconductor heterostructure where the metallic electrostatic gates are replaced by a patterned ferroelectric material. The structure consists of an array of Ge dots embedded in a Si matrix capped with a patterned uniaxial ferroelectric oxide having the spontaneous polarization pointing perpendicular to the plane of the dots ( $c$-axis oriented) (figure 8 ). The electron spin qubits are initialized by optically exciting electron-hole pairs in the quantum dots using circularly polarized light. Due to the type II band alignment between the strained $\mathrm{Ge}$ and the $\mathrm{Si}$, the electron-hole pair will spatially separate with the hole tightly confined in the Ge dot and the electron confined in the Si matrix. The electron remains localized close to the parent dot both due to the weak exciton binding energy as well as due the electrostatic potential produced by the bound charges in the ferroelectric. 


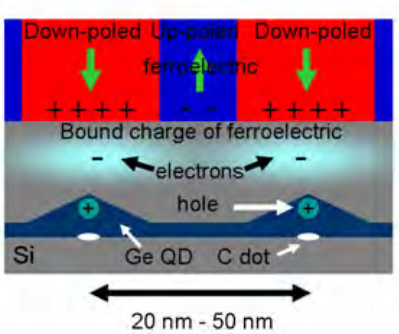

(a)

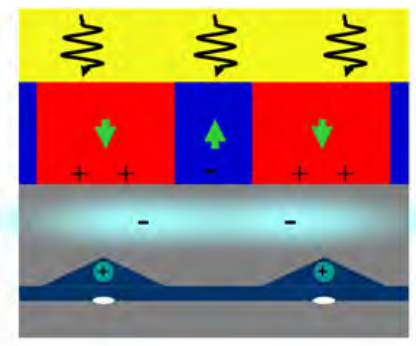

(b)

Figure 8. (a) Ge quantum dots (QD) embedded in a Si host capped with an oxide ferroelectric material. Due to the band alignment the electrons are confined in the Si matrix, while the holes are confined in the $\mathrm{Ge}$; (b) optical excitation modulates the ferroelectric polarization resulting in a modulation of the electrons exchange coupling.

While the static polarization of the ferroelectric material is used to assist with the confinement of the electrons, electro-optic modulation of the ferroelectric polarization can be used to modulate the Heisenberg exchange interaction between electrons localized at different quantum dots. For laser pulses with a time-dependent optical intensity $I_{\mathrm{opt}}(t)$ the nonlinear optical polarization is given by

$$
P^{(2)}(t)={ }_{2 c}^{r n^{3}} I_{\mathrm{opt}}(t)
$$

where $r$ is the electro-optic coefficient, $n$ is the refractive index and $c$ is the speed of light. For average optical powers of $10 \mathrm{~mW}$ focused to a diffraction limited spot on ferroelectrics such as $\mathrm{BaTiO}_{3}$, one obtains [33] values for the nonlinear polarization: $P^{(2)}(t)=6.29 \times$ $10^{-2} \mu \mathrm{C} \mathrm{cm}^{-2}$. While such large optical powers might raise concerns regarding the undesired photogeneration of carriers, these issues are addressed through non-resonant optical gating. Tuning of both the optical pulse duration and strength allows full control over the exchange interaction through dynamic ferroelectric polarization at terahertz frequencies.

Considering the small separation of the dots for sufficient wavefunction overlap, the spatial resolution of optical probes determined by diffraction might impose constraints on the proposal. Spin cluster approaches developed for the implementation of fast 'exchange-only' universal computation $[71,73]$ offer one way to increase the spatial extent of a spin qubit. Figure 9 shows a spin cluster containing nine spins coupled by a static Heisenberg exchange coupling. The ground state of this system is twofold degenerate and can be used as a spatially extended qubit. For optically accessing individual quantum dots and controlling their interactions, Imamoglu et al [91] have proposed an alternate way to couple localized electron spins using cavity quantum electrodynamics. A recent demonstration of tuning nanocavity modes with singlequantum-dot excitons was demonstrated for an InAs/GaAs quantum dot system [92].

One of the main challenges for the Ge dots on Si quantum computation scheme is the optical initialization of the electrons through momentum transfer from circularly polarized light to spin polarized electrons. In zincblende semiconductors initialization was achieved due to favourable optical selection rules [93], but it remains to be demonstrated for Si structures, since both $\mathrm{Si}$ and $\mathrm{Ge}$ are indirect semiconductors requiring phonon assistance for absorption and emission of photons. Due to lattice strain, epitaxial growth of Ge on Si leads to the formation of self-assembled islands. However, their size $(20-100 \mathrm{~nm})$ is too large to be of use for confining single electrons, or for photoexcitation of spin polarized electrons. The transition from indirect to direct character for $\mathrm{Ge} / \mathrm{Si}$ quantum dots is expected to occur for sizes below 


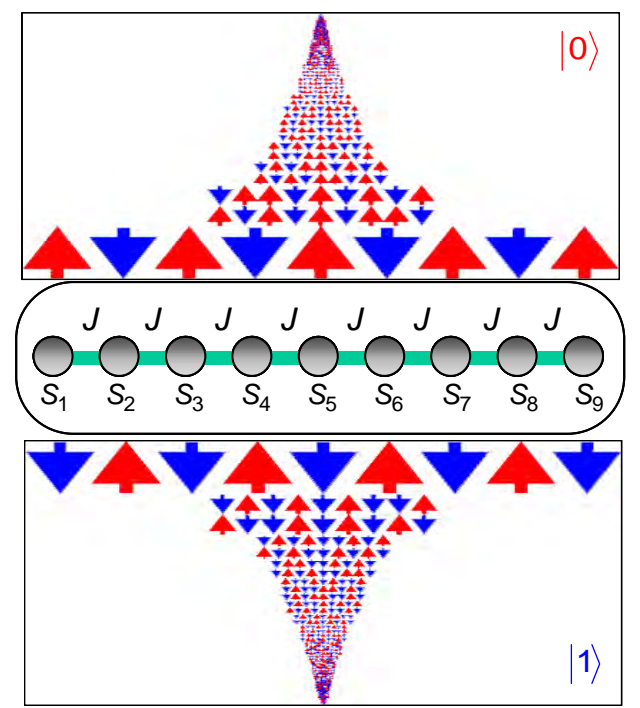

Figure 9. Spin cluster qubit state scales up the spin wavefunction to the wavelength of light. A chain of nine electron spins are coupled by nearest neighbour Heisenberg exchange. The ground state is twofold degenerate and can be used as a stable qubit.

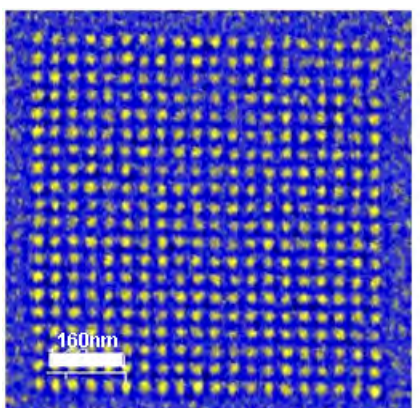

(a)

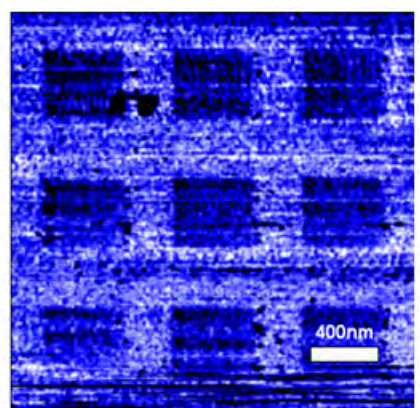

(b)

Figure 10. (a) AFM image of $20 \times 20$ array of $20 \times 20$ array of $\mathrm{Ge}$ islands with diameters $<10 \mathrm{~nm}$; (b) Ferroelectric domain writing on a $100 \AA \mathrm{BaTiO}_{3} / 300 \AA(\mathrm{Ba}, \mathrm{Sr}) \mathrm{TiO}_{3} / \mathrm{Si}(100)$ thin film.

$10 \mathrm{~nm}$ [94]. For a strained Ge dot on Si quantum dot with $8 \mathrm{~nm}$ in diameter and $2 \mathrm{~nm}$ in height Pryor et al [95] have calculated a band gap of $0.5 \mathrm{eV}$ and a $83 \%$ spin polarization degree for excitation with circularly polarized light. Recently, Guise et al [96] have succeeded in developing a method for templated growth of sub-10 nm Ge dots on Si (figure 10(a)). Carbon deposition at specific location using high resolution electron beam dissociation of hydrocarbons molecules and subsequent annealing, templates the Si surface with a predetermined strain profile favourable to the formation of Ge small islands at specific locations.

From a materials point of view, the growth of properly oriented ferroelectrics on Si remains a challenge. Strain control through a specially designed relaxed $(\mathrm{Ba}, \mathrm{Sr}) \mathrm{TiO}_{3}$ buffer layer on (001) Si has been recently shown to allow growth of $c$-axis (perpendicular to the substrate) oriented $\mathrm{BaTiO}_{3}$ thin films [97]. Ferroelectric domain patterning has been demonstrated on films as thin as $100 \AA$, with controllable reversal of the polarization direction (figure 10(b)). 
While as described above for SiGe based quantum dot proposals for quantum computation, many material issues have been addressed, functioning fundamental components relevant to quantum computation, such as qubits and quantum gates, remain to be demonstrated.

\section{Prospects for high temperature operation}

\section{Electron qubits associated with deep donor impurities}

The requirement for very low temperature operation in most solid state computations schemes stems partly from the necessity to take advantage of the longer spin coherence times. For proposals employing shallow donors, high temperature operation would lead to ionization effects due to the weak coupling of the electrons with the parent donor. Trying to eliminate these constraints, Stoneham et al $[98,99]$ have proposed the use as a qubit representation of electron spins associated with deep donors, such as $\mathrm{Bi}$ in $\mathrm{Si}$, which are not as easily ionized by thermal excitation. One original feature of the scheme proposed is to control the two-qubit interaction using a third control impurity atom with a different energy spectrum, such as Er. Optical excitation promotes an electron from the control atom in an excited state coupled with the ground states of both qubit atoms. Qubit-qubit interactions are optically switched on/off by optical excitation and stimulated de-excitation of the control electron, without the need of electrostatic gates. The proposal takes advantage of the natural occurrence of disordered arrangements of donors to address the issue of limited spatial resolution of optical probes when compared with the 5-10 nm separation between donors required for sufficient wavefunction overlap. Spectral selectivity of non-equivalent control atoms is used to access specific qubit pairs. Since the quantum information is always stored in the ground state of the deep donors, the energy scales involved in the qubit manipulation are large, leading Stoneham et al [98] to suggest the possibility of room temperature operation.

\section{Nitrogen vacancies in carbon systems}

The long decoherence times measured in silicon based systems are exceeded in the solid state environments only by those in carbon based material systems. In particular, the electronic states associated with nitrogen vacancy (NV) defect centres in diamond (figure 11(a)) show coherence times $T_{2}$ of $2 \mathrm{~s}$ at $T=1.5 \mathrm{~K}$ [100] and up to $60 \mu \mathrm{s}$ [101] at room temperature. The reasons for this behaviour are related with low atomic mass and implicit smaller spin-orbit couplings, along with a high natural occurrence of spin-zero isotopes (99\%) without further isotopic purification. These characteristics make carbon an attractive system for the possible implementation of simple quantum algorithms. The nuclear spins of $\mathrm{C}^{13}$ coupled through the hyperfine interaction with such electron paramagnetic NV centres in diamond, have also been proposed as qubits [100]. For an electron spins delocalization of $\sim 4 \AA$ [102] associated with these centres, the number of nuclei with which they couple is relatively large. Because of the non-equivalent coupling with different $\mathrm{C}^{13}$ nuclei within the diamond lattice, the individual qubits become distinguishable by monitoring by optical spectroscopy the hyperfine splitting of the transitions between the first electronic exited state ${ }^{3} \mathrm{E}$ and the triplet $(S=1)$ ground state ${ }^{3} \mathrm{~A}$ (figure $11(\mathrm{~b})$ ). The specific optical excitation spectrum of the nitrogen vacancy defects can also be used to spin polarize the ground state using the metastable single state ${ }^{1} \mathrm{~A}$, since relaxation from this state does not conserve the spin. The gating of the qubits is realized through a standard NMR procedure with microwave pulses. Recently, Jelezko et al [103] have implemented two-qubit conditional gates (CROT gate) with the two bits being a single electron spin and a single ${ }^{13} \mathrm{C}$ nuclear spin. The demonstrated speed of $0.1 \mu \mathrm{s}$ of the CROT gate indicates that up to $10^{3}$ operations at room temperature are already attainable. Combined 


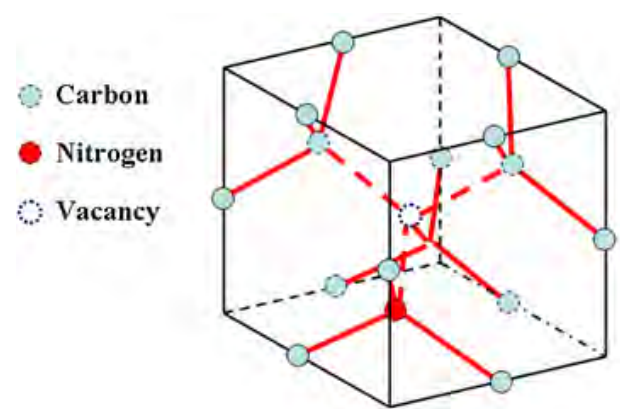

(a)

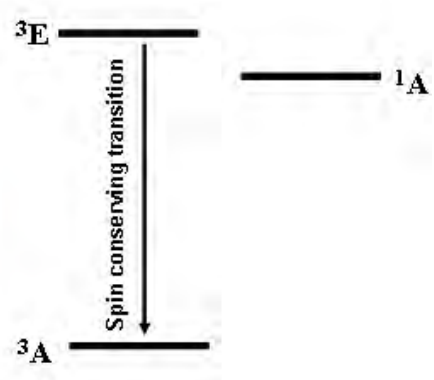

(b)

Figure 11. (a) NV centre in a diamond lattice; (b) schematic diagram of the NV defect centre energy levels $\left({ }^{1} \mathrm{~A}\right.$ is a metastable singlet state while ${ }^{3} \mathrm{E}$ and ${ }^{3} \mathrm{~A}$ are triplet states).

with advances in controlled patterning of NV centres through ion beam implantation currently limited to about $1 \mu \mathrm{m}$ accuracy [104], these results represent an interesting development for diamond based quantum computer implementations.

One of the biggest challenges for spins in diamond quantum computation implementations is coupling spins localized at different sites. Coherent optical coupling of two molecules separated by $12 \mathrm{~nm}$ in an organic crystal has been proposed and achieved [105], but controlled entanglement at two distinct sites remains to be demonstrated.

\section{The measurement problem}

Independently of the particular choice of qubit and qugate, any device for measuring the state of a qubit must meet certain requirements in terms of speed and sensitivity. First, measurements must take place on a time scale shorter than the qubit decoherence time in order to enable quantum error correction codes to be implemented. Second, coupling between the measuring device and the system has to be sufficiently weak, outside the measurement interval, in order to avoid state mixing. While some of the most promising proposals take advantage of the natural two-level spin system of the electrons and spin-1/2 nuclear spins, direct measurement of the magnetic moment of single spins is a challenging task. The sensitivity of conventional electron spin resonance (ESR) and nuclear magnetic resonance (NMR) is limited to about $10^{7}$ electron spins [106] and $10^{12}$ nuclear spins [107], respectively. Nevertheless, a couple of approaches that combine standard magnetic resonance techniques with atomic force or optical microscopy have been able to measure single-electron magnetic states and the corresponding hyperfine interaction coupled nuclear states, showing promise for quantum computation related applications. Rugar et al [108] have used magnetic resonance force microscopy (MRFM) to detect a single electron spin with a spatial resolution of $25 \mathrm{~nm}$. The technique, based on Sidles's original proposal ten years prior [109], employs a magnetic particle attached to the end of a cantilever to perturb the local spin resonance condition in the presence of a uniform and a microwave magnetic field. MRFM techniques have been proposed not only for read-out of the qubit states but also for the initialization and gating process using quantum information transfer from the localized qubits to the magnetic particle [48]. As described in section 2.2, the relatively high sensitivity of this method has been addressed by Ladd et al [66], who proposed the embedding of the quantum computing structure consisting of $\mathrm{Si}^{29}$ chains in a silicon bridge integrated with micromagnets. The magnetization state of up to $10^{5}$ equivalent $\mathrm{Si}^{29}$ nuclear spins can be determined from the phase of the bridge oscillations, thus increasing the sensitivity proportionally. With the observation of ESR for electrons associated with impurities in 
Si [110], spin-dependent tunnelling from an STM probe has also been proposed [111], with the added benefit of avoiding the decoherence effects introduced in MRFM by the coupling between the ferromagnetic particle and the spin system.

Optically detected magnetic resonance (ODMR) used for spin detection in organic molecules is also a well-established technique [112]. In the case of ODMR the information about the spin system is contained in the intensity of luminescence which is dependent on the population of specific spin levels. Using this technique, coherent oscillation of a single nuclear magnetic spin associated with a nitrogen vacancy in diamond has been observed [103]. ODMR has also been proposed for measuring single spin states in semiconductor quantum dots, taking advantage of the Pauli blocking principle to induce selective optical excitation [113].

Some of the most extensively used and successful optical probes for measuring the spin dynamics in semiconductor structures are based on the Faraday rotation effect in which the polarization of a linearly polarized probe beam is rotated by an amount proportional to the magnetic moment [114]. The highest reported sensitivity for III-V semiconductors is 100 spins $\mathrm{Hz}^{-1 / 2}$ [115]. While, for such optical probes the quantum efficiency is small, a notable characteristic is their spectral selectivity. The currently unavoidable device variability resulting in specific energy spectra for each qubit, may thus be used to measure them individually using efficient multi-channel spectrally resolved phase sensitive detection [116] or using narrow bandwidth laser probes combined with high speed detection electronics [117].

While the above-mentioned probes can provide information about the state of single qubits, it is not evident how they can be scaled for systems with large number of qubits. This difficulty of measuring single spins and addressing the issue of scalability, ideally in-line with the existing electronic industry infrastructure, was recognized early in the development of proposals for quantum computation in a solid state environment, and alternative routes to the direct spin measurement have been proposed [43]. While charge qubits lack the long decoherence times associated with spin systems, the technology for measuring charge is much better developed. Single-electron transistors (SET) [118] can routinely measure sub-electron charge variations. Radio-frequency SET have demonstrated charge sensitivities of $1.2 \times 10^{-5} \mathrm{e} \mathrm{Hz}^{-1 / 2}$ with a constant gain from dc to better than $100 \mathrm{MHz}$, and a recent theoretical analysis indicates that sensitivity values as high as $\sim 2 \times 10^{-8} e \mathrm{~Hz}^{-1 / 2}$ are possible [119]. In order to take advantage both of the increased coherence in spin systems and the sensitivity of charge measurements, various schemes for spin-to-charge conversion have been proposed. For qubits formed by shallow donors in Si, Kane [120] proposes to use RF-SET to monitor the spin-dependent tunnelling between neighbouring $\mathrm{P}^{31}$ donor impurities. The exchange splitting between singlet and triplet states may be detectable using a SET. The use of SET to infer the spin state of a system from charge movements is not limited to quantum computing architectures based on donor impurities, but can also be extended to quantum dot systems which are characterized by similar discrete spectra. Friesen et al [121] have proposed rapid spin initialization and read-out based the use of SET to detect spin-dependent oscillations of an electron in an asymmetric dot driven by microwave oscillations.

Additional methods based on the use of spin filters that transmit electrons with defined spin orientations have been described in a recent review [122]. Currently, these methods have been very successful in GaAs based systems, where a single electron spin state in a quantum dot has been measured [82], and as the material issues in group IV materials are resolved they can be extended into this field.

\section{Conclusions}

We have described various approaches based on group IV materials for the implementation of a solid state quantum computer. At present, there is no clear indication of which implementation 
appears to be most promising. Nevertheless, the large coherence times both for electrons and nuclei and the potential easy integration with the current electronic technology makes these materials very attractive for the quantum computation purposes. In recent years many of the material science issues regarding accurate control over $\mathrm{Si}-\mathrm{Ge}$ nanostructure fabrication and donor positioning have been resolved. The main challenge at hand remains the demonstration of single-and two-qubit gates and the implementation of functioning simple quantum algorithms in these solid state environments. It also remains to be seen whether group IV based approaches (and solid state approaches in general) will live up to their promise of being able to deliver scalability, once the challenges at the one-qubit and two-qubit level have been satisfactorily resolved.

\section{Acknowledgments}

The authors are grateful to Patrick Irvin for a careful reading of the manuscript. This work was supported by DARPA (DAAD 19-01-1-0650).

\section{References}

[1] Feynman R 1985 Quantum Mech. Comput. Opt. News 1111

[2] Hopcroft J and Ullman J 1979 Introduction to Automata Theory, Languages and Computation (Reading, MA: Addison-Wesley)

[3] Deutsch D 1985 Proc. R. Soc. A 40097

Deutsch D 1997 The Fabric of Reality (London: Penguin)

Aspuru-Guzik A, Dutoi A D, Love P J and Head-Gordon M 2005 Science 3091704

[4] Moore G 1965 Electronics 388

[5] Deutsch D and Jozsa R 1992 Proc. R. Soc. A 439553

[6] Shor P W 1994 Proc. 35th Symp. on the Foundations of Computer Science p 124 Shor P W 1997 SIAM J. Sci. Stat. Comput. 361484

[7] Gerjuoy E 2005 Am. J. Phys. 73521

[8] Beauregard S 2003 Quantum Inf. Comput. 3175 Gossett P 1998 Preprint quant-ph/9808061 Zalka C 1998 Proc. R. Soc. A 454313

[9] http://www.rsasecurity.com

[10] Grover L K 1997 Phys. Rev. Lett. 79325

[11] Bennett C H, Bernstein E, Brassard G and Vazirani U 1997 SIAM J. Comput. 261510 Simon D 1997 SIAM J. Comput. 261474 Vazirani U 1998 Phil. Trans. R. Soc. A 3561759

[12] DiVincenzo D P 1995 Phys. Rev. A 511015

Barenco A, Bennett C H, Cleve R, DiVincenzo D P, Margolus N, Shor P, Sleator T, Smolin J A and Weinfurter H 1995 Phys. Rev. A 523457

[13] DiVincenzo D P 2000 Fortschr. Phys. 48771

[14] Nielsen M A and Chuang I L 2000 Quantum Computation and Quantum Information (Cambridge: Cambridge University Press)

[15] Kane B E 2005 MRS Bull. 30105

[16] Haroche S and Raimond J-M 1996 Phys. Today 8 (August) 51 Dyakonov M I 2001 Preprint cond-mat/0110326

[17] Landauer R 1996 Phys. Lett. A 217188

[18] Calderbank A R and Shor P W 1996 Phys. Rev. A 541098 Steane A M 1996 Phys. Rev. Lett. 77793

[19] Preskill J 1998 Nature 391631

[20] Preskill J 1998 Proc. R. Soc. A 454385

[21] Preskill J 1998 Proc. R. Soc. A 454469

[22] Steane A M 1996 Phys. Rev. Lett. 782252

[23] DiVincenzo D P and Loss D 1998 Superlatt. Microstruct. 23419 
[24] Shannon C E (ed) 1998 The Mathematical Theory of Information (Urbana, IL: University of Illinois Press) (1949 reprinted)

[25] Gershenfeld N A and Chuang I L 1997 Science 275350

[26] Domokos P, Raimond J M, Brune M and Haroche S 1995 Phys. Rev. A 523354

[27] Cirac J I and Zoller P 1995 Phys. Rev. Lett. 744091

[28] Takeuchi S 2000 Phys. Rev. A 62032301

Kwiat P G, Mitchell J R, Schwindt P D D and White A G 2000 J. Mod. Opt. 47257

[29] Loss D and DiVincenzo D 1998 Phys. Rev. A 57120

[30] Kane B E 1998 Nature 393133

[31] Vrijen R, Yablonovitch E, Wang K, Jiang H W, Baladin A, Roychowdhury V T M and DiVincenzo D 2000 Phys. Rev. A 62012306

[32] Friesen M, Rugheimer P, Savage D E, Lagally M G, van der Weide D W, Joynt R and Eriksson M A 2003 Phys. Rev. B 67121301

[33] Levy J 2001 Phys. Rev. A 64052306

[34] Barenco A, Deutsch D, Ekert A and Jozsa R 1995 Phys. Rev. Lett. 744083

[35] Gordon J P and Bowers K D 1958 Phys. Rev. Lett. 1368

[36] Saykin S, Mozyrsky D and Privman V 2002 Nano Lett. 2651

[37] Tyryshkin A M, Lyon S A, Astashkin A V and Raitsimring A M 2003 Phys. Rev. B 68193207

[38] Kikkawa J M and Awschalom D D 1998 Phys. Rev. Lett. 804313

[39] Koppens F H L, Folk J A, Elzerman J M, Hanson R, Beveren L H W v, Vink I T, Tranitz H P, Wegscheider W, Kouwenhoven L P and Vandersypen L M K 2005 Science 3091346

[40] Feher G 1959 Phys. Rev. 1141219

Wilson D K and Feher G 1961 Phys. Rev. 1241068

[41] Clark R G, Brenner R, Buehler T M, Chan V, Curson N J, Dzurak A S, Gauja E, Goan H S, Greentree A D, Hallam T, Hamilton A R, Hollenberg L C L, Jamieson D N, McCallum J C, Milburn G J, O’Brien J L, Oberbeck L, Pakes C I, Prawer S D, Reilly D J, Ruess F J, Schofield S R, Simmons M Y, Stanley F E, Starrett R P, Wellard C and Yang C 2003 Phil. Trans. R. Soc. 3611451

[42] Hollenberg L C L, Dzurak A S, Wellard C, Hamilton A R, Reilly D J, Milburn G J and Clark R G 2003 Preprint cond-mat/0306235

[43] Kane B E 2000 Fortschr. Phys. 489

[44] Hayashi T, Fujisawa T, Cheong H D, Jeong Y H and Hirayama Y 2003 Phys. Rev. Lett. 91226804

[45] Gorman J 2005 Phys. Rev. Lett. 95090502

[46] Lampel G 1968 Phys. Rev. Lett. 20491

[47] Boykin P O, Mor T, Roychowdhury V, Vatan F and Vrijen R 2002 Proc. Natl Acad. Sci. 993388

[48] Berman G P, Doolen G D, Hammel P C and Tsifrinovich V I 2000 Phys. Rev. B 6114694

[49] Fiederling R, Keim M, Reuscher G, Ossau W, Schmidt G, Wang A and Molenkamp L W 1999 Nature 402787 Ohno Y, Young D K, Beschoten B, Matsukura F, Ohno H and Awschalom D D 1999 Nature 402790

[50] Koiller B, Hu X and Sarma S D 2002 Phys. Rev. Lett. 88027903

[51] Sarma S D, Sousa R, Hu X and Koiller B 2005 Solid State Commun. 133737

[52] Koiller B, Hu X and Sarma S D 2002 Phys. Rev. B 66115201

[53] O’Brien J L, Schofield S R, Simmons M Y, Clark R G, Dzurak A S, Curson N J, Kane B E, McAlpine N S, Hawley M E and Brown G W 2002 Smart Mater. Struct. 11741

Tucker J R and Shen T C 1998 Solid State Electron Devices 421061

Shen T-C, Wang C, Abeln G C, Tucker J R, Lyding J W, Avouris P and Walkup R E 1995 Science 2681590

[54] Schofield S R, Curson N J, Simmons M Y, Rueb F J, Hallam T, Oberbeck L and Clark R G 2003 Phys. Rev. Lett. 91136104

[55] Ruess F J, Oberbeck L, Simmons M Y, Goh K E J, Hamilton A R, Hallam T, Schofield S R, Curson N J and Clark R G 2004 Nano Lett. 41969

[56] Shen T-C, Kline J S, Schenkel T, Robinson S J, Ji J Y, Yang C, Du R R and Tucker J R 2004 J. Vac. Sci. Technol. B 223182

[57] Oberbeck L, Curson N J, Hallam T, Simmons M Y, Bilger G and Clark R G 2004 Appl. Phys. Lett. 851359

[58] Schenkel T, Persaud A, Park S J, Nilsson J, Bokor J, Liddle J A, Keller R, Schneider D H, Cheng D W and Humphries D E 2003 Appl. Phys. Lett. 947017

[59] Sousa R d, Delgado J D and Sarma S D 2004 Phys. Rev. A 70052304

[60] Mozyrsky D, Privman V and Glasser M L 2001 Phys. Rev. Lett. 865112 Privman V, Vagner I D and Kventsel G 1998 Phys. Lett. A 239141

[61] Mozyrsky D, Privman V and Vagner I D 2001 Phys. Rev. B 63085313

[62] Skinner A J, Davenport M E and Kane B E 2003 Phys. Rev. Lett. 90087901 
[63] Kikkawa J M and Awschalom D D 1999 Nature 397139

[64] Eng K, McFarland R N and Kane B E 2005 Appl. Phys. Lett. 87052106

[65] Hollenberg L C L, Greentree A D, Fowler A G and Wellard C J 2005 Preprint quant-ph/0506198

[66] Ladd T D, Goldman J R, Yamaguchi F, Yamamoto Y, Abe E and Itoh K M 2002 Phys. Rev. Lett. 89017901

[67] Abe E, Itoh K M, Ladd T D, Goldman J R, Yamaguchi F and Yamamoto Y 2003 J. Supercond. 16175

[68] Ladd T D, Maryenko D, Yamamoto Y, Abe E and Itoh K M 2005 Phys. Rev. B 71014401

[69] Itoh K M 2005 Solid State Commun. 133747

[70] Martin A S, Capaz R B and Koiller B 2004 Phys. Rev. B 69085320

[71] DiVincenzo D P, Bacon D, Kempe J, Burkard G and Whaley K B 2000 Nature 408339

[72] Weinstein Y S, Hellberg C S and Levy J 2005 Phys. Rev. A 72020304 Raussendorf R, Browne D E and Briegel H J 2003 Phys. Rev. A 68022312

[73] Levy J 2002 Phys. Rev. Lett. 89147902

[74] Yablonovitch E, Jiang H W, Kosaka H, Robinson H D, Rao D S and Szkopek T 2003 Proc. IEEE 91761

[75] Kato Y, Myers R C, Driscol D, Gossard A C, Levy J and Awschalom D D 2003 Science 2991201

[76] Kato Y, Myers R C, Gossard A C and Awschalom D D 2004 Nature 42740

[77] Khaetskii A V and Nazarov Y V 2000 Phys. Rev. B 6112639

[78] Johnson A T, Kouwenhoven L P, de Jong W, van der Vaart N C, Harmans C J P M and Foxon C T 1992 Phys. Rev. Lett. 691592

[79] Fafard S, Leon R, Leonard D, Merz J L and Petroff P M 1995 Appl. Phys. Lett. 661767

[80] Tarucha S, Austing D G, Honda T, van der Hage R J and Kouwenhoven L P 1996 Phys. Rev. Lett. 773613

[81] Ribeiros G M, Leonard D and Petroff P M 1995 Appl. Phys. Lett. 661767

[82] Elzerman J M, Hanson R, Beveren L H W v, Witkamp B, Vandersypen L M K and Kouwenhoven L P 2004 Nature 430431

[83] Kroutvar M, Docummun Y, Heiss D, Bichler M, Schuh D, Abstreiter G and Finley J J 2004 Nature 43281

[84] Dutt M V G, Cheng J, Li B, Xu X, Li X, Berman P R, Steel D G, Bracker A S, Gammon D, Economou S E, Liu R-B and Sham L J 2005 Phys. Rev. Lett. 94227403

[85] Burkard G, Loss D and DiVincenzo D 1999 Phys. Rev. B 592070

[86] Mooney P M 1996 Mater. Sci. Eng. R 17105 Ismail K 1996 J. Vac. Sci. Technol. B 142776

[87] Klein L J, Lewis K L M, Slinker K A, Goswami S, van der Weide D W, Blick R H, Mooney P M, Chu J O, Coppersmith S N, Friesen M and Eriksson M A 2005 Preprint cond-mat/0503766

[88] Slinker K A, Lewis K L M, Haselby C C, Goswami S, Klein L J, Chu J O, Coppersmith S N, Joynt R, Blick R H, Friesen M and Eriksson M A 2005 Preprint cond-mat/0508107

[89] Yilmaz T, DePriest C M and Delfyet P J 2001 Electron. Lett. 371338

[90] Levy J 2002 Phys. Status Solidi b 233467

[91] Imamoglu A, Awschalom D D, Burkard G, DiVincenzo D P, Loss D, Sherwin M and Small A 1999 Phys. Rev. Lett. 834205

[92] Badolato A, Hennessy K, Atature M, Dreiser J, Hu E, Petroff P M and Imamoglu A 2005 Science 3081158

[93] Crooker S A, Baumberg J J, Flack F, Samarth N and Awschalom D D 1997 Phys. Rev. B 567574

[94] Takagahara T and Takeda K 1992 Phys. Rev. B 4615578

[95] Pryor C E and Flatte M E 2003 unpublished

[96] Guise O, Yates J T, Ahner J, Vaithyanathan V and Schlom D G 2005 Appl. Phys. Lett. 87171902

[97] Vaithyanathan V, Lettieri J, Tian W, Kochhar A, Ma H, Sharan A, Vasudevarao A, Gopalan V, Li Y, Chen L Q, Zschack P, Woicik J C, Levy J and Schlom D G 2005 unpublished

[98] Stoneham A M, Fisher A J and Greenland P T 2003 J. Phys.: Condens. Matter 15 L447

[99] Rodriguez R, Fisher A J, Greenland P T and Stoneham A M 2004 J. Phys.: Condens. Matter 162757

[100] Kilin S Y and Wrachtrup J 2000 Erwin Schrödinger International Institute for Mathematical Physics Preprints p 1 http://www.esi.ac.at/preprints/ESI-Preprints.html (Posting No. 950)

[101] Kennedy T A, Colton J S, Butler J E, Linares R C and Doering P J 2003 Appl. Phys. Lett. 834190

[102] Luszczeka M, Laskowskib R and Horodeckia P 2004 Physics B 348292

[103] Jelezko F, Gaebel T, Popa I, Domhan M, Gruber A and Wrachtrup J 2004 Phys. Rev. Lett. 93130501

[104] Martin J, Wannemacher R, Teichert J, Bishoff L and Koehler B 1999 Appl. Phys. Lett. 753096

[105] Hettich C, Schmitt C, Zitzmann J, Kuhn S, Gerhardt I and Sandoghdar 2002 Science 298385

[106] Blank A, Dunnam C R, Borbat P P and Freed J H 2003 J. Magn. Reson. 165116

[107] Ciobanu L, Seeber D A and Pennington C H 2002 J. Magn. Reson. 158178

[108] Rugar D, Budakian R, Mamin H J and Chui B W 2004 Nature 430329

[109] Sidles J A, Garbini J L, Bruland K J, Rugar D, Zuger O, Hoen S and Yannoni C S 1995 Rev. Mod. Phys. 67249

[110] Manassen Y, Mukhopadhyay I and Rao N R 2000 Phys. Rev. B 6116223 
[111] Berman G P, Brown G W, Hawley M E and Tsifrinovich V I 2001 Phys. Rev. Lett. 87097902

[112] Jelezko F and Wrachtrup J 2004 J. Phys.: Condens. Matter 16 R1089

[113] Gywat O, Engel H-A, Loss D, Epstein R J, Mendoza F M and Awschalom D D 2004 Phys. Rev. B 69205303

[114] Sih V A, Johnston-Halperin E and Awschalom D D 2003 Proc. IEEE 91752

[115] Kato Y K, Myers R C, Gossard A C and Awschalom D D 2004 Phys. Rev. Lett. 93176601

[116] Fodor P S, Rothenberger S and Levy J 2005 Rev. Sci. Instrum. 76013103

[117] Irvin P, Fodor P S and Jevy J 2005 unpublished

[118] Averin D V and Likharev K K 1987 J. Low Temp. Phys. 62345 Fulton T A and Dolan G J 1987 Phys. Rev. Lett. 59109

[119] Rao D S, Szkopek T, Jiang H W and Yablonovitch E 2005 Preprint quant-ph/0507224

[120] Kane B E, McAlpine N S, Dzurak A S, Clark R G, Milburn G J, Sun H B and Wiseman H 2000 Phys. Rev. B 612961

[121] Friesen M, Tahan C, Joynt R and Eriksson M A 2004 Phys. Rev. Lett. 92037901

[122] Cerletti V, Coish W A, Gywat O and Loss D 2005 Nanotechnology 16 R27

Post-print standardized by MSL Academic Endeavors, the imprint of the Michael Schwartz Library at Cleveland State University, 2017. 